\title{
Estimação da influência de variáveis macroeconômicas sobre o faturamento de organizações siderúrgicas usando o ARMAX
}

\section{Estimating the influence of macroeconomic variables on steel sales organizations using ARMAX}

\author{
Pedro H. M. Albuquerque ${ }^{1}$ \\ Letícia da Costa e Silva ${ }^{1}$ \\ Yuri Sampaio Maluf ${ }^{1}$
}

\begin{abstract}
Resumo: Este artigo utiliza a metodologia ARMAX para a previsão de faturamento de empresas inseridas na indústria siderúrgica nacional. $\mathrm{O}$ artigo buscou evidenciar as relações existentes entre os faturamentos futuros contra informações macroeconômicas bem como os próprios dados defasados das receitas das companhias siderúrgicas. A análise foi empregada no período do segundo trimestre de 2002 ao último de 2010 e compreendeu quatro empresas: CSN, Gerdau, Usiminas e Vicunha. Para cada uma das tais companhias, foram estimadas 6.480 estruturas distintas, sendo selecionadas as que detinham os melhores critérios de informação (AIC). O estudo mostra que, de fato, os dados passados são relevantes para as estimativas futuras dos faturamentos das companhias. Além disto, a principal variável exógena do ARMAX foi a taxa de juros, representada pela SELIC, seguida do PIB e da inflação. A partir desta dinâmica, é possível auxiliar gestores, investidores e analistas de mercado para melhor gerenciamento de seus ativos. Palavras-chave: ARMAX. Previsão do faturamento. Fatores macroeconômicos. Siderurgia.
\end{abstract}

\begin{abstract}
This research applied ARMAX methodology for forecasting the revenue of companies in the Brazilian steel industry market. Thus, the article sought to highlight the relationship between future revenues through the data of interest rates, economic growth and inflation rate, as well as its own revenue lagged. Analysis was employed with four companies: CSN, Gerdau, Usiminas and Vicunha. The sample period extended from the second quarter of 2002 to the last quarter of 2010. For each of these companies, 6480 different structures were estimated, and those containing the best information criteria $(A I C)$ were selected. The study shows that, in fact, the past data are relevant to predict the future revenues of enterprises. Moreover, the main exogenous variable of ARMAX was interest rate - represented by the SELIC rate, followed by GDP and inflation rate. This dynamics could help managers, investors and market analysts to manage their assets better.
\end{abstract}

Keywords: ARMAX. Revenue forecast. Macroeconomic factors. Steel industry.

\section{Introdução}

O faturamento é vital para as companhias. Objetivos e recursos são as palavras-chave na definição de administração e organização. O conceito de recursos engloba pessoas, recursos materiais, espaço, instalação, máquinas, móveis, equipamentos e também recursos intangíveis como tempo e conhecimento (MAXIMIANO, 2004).

Maximiano (2004) relata ainda que as organizações que mensuram seus níveis de susceptibilidades a determinados dados econômicos permitem se preparar e minimizar seus riscos. Segundo o autor, a possibilidade de prever, em especial as receitas, pode acarretar benefícios como a análise dos objetivos, planejamento do uso do tempo, planejamento dos recursos, avaliação dos riscos e previsão dos meios de controle.

A siderurgia brasileira teve seu início em 1557 , com a instalação de uma pequena produtora de ferro no Estado de São Paulo. A partir disto, muitas indústrias do ramo vieram para o Brasil e, com o surgimento da Companhia Siderúrgica Belgo-Mineira em 1921, o setor passou a se desenvolver com mais expressividade. No entanto, o grande marco para a siderurgia foi à criação da Companhia Siderúrgica Nacional (CSN) em 1946, que começou a operar a maior usina produtora de aço da América Latina. A

${ }^{1}$ Departamento de Administração, Instituto Central de Ciências - ICC, Universidade de Brasília - UnB, Campus Universitário Darcy Ribeiro, Ala Norte, Bloco B, $1^{\circ}$ Andar, Sala 576, CEP 70910-900, Brasília, DF, Brasil, e-mail: pedroa@ unb.br;

lelscs@yahoo.com.br; yurimaluf@gmail.com

Recebido em 19/9/2012 — Aceito em 29/5/2014

Suporte financeiro: Nenhum. 
CSN foi também a primeira empresa a produzir aços planos, laminados a quente e a frio e em revestidos (ANDRADE; CUNHA, 2002).

Durante o início dos anos 40, o processo de industrialização continuou, foram criadas: a Companhia Vale do Rio Doce (1942), a Fábrica Nacional de Motores (1943) e a Companhia Álcalis (1943). Porém, a segunda metade da década foi marcada pela estagnação dos empreendimentos em função do pós Segunda Guerra Mundial (GREMAUD; TORNETO JUNIOR; VASCONCELLOS, 2005).

De acordo com dados divulgados no sítio eletrônico do Instituto Aço Brasil, o País conta com vinte e nove unidades industriais produtoras de aço administradas por onze grupos empresariais. Segundo estudo realizado pela Fundação Getúlio Vargas (FGV), em 2010, o aço foi responsável por um total de R \$ 146,8 bilhões, ou 4,0\%, do produto interno bruto do País. Em 2011, o Brasil produziu 35,2 milhões de toneladas de Aço Bruto e o setor registrou cerca de 60 mil empregados efetivos no final do mesmo ano (INSTITUTO..., 2013).

Quanto maior a empresa, maiores são as suas necessidades de recursos e, consequentemente, maiores serão as suas despesas e o faturamento que ela necessita para se sustentar. Sendo assim, a possibilidade de prever o faturamento apresenta-se como um importante instrumento para o dimensionamento de seus indicadores e, consequentemente, pode trazer vantagens competitivas como melhorias na gestão e alocação de recursos de forma otimizada. A econometria é uma das possibilidades para a construção de modelos de previsão.

A econometria é baseada no desenvolvimento de métodos estatísticos para estimar relações econômicas, testar teorias, avaliar e implementar políticas de governo e de negócios. A aplicação mais comum da econometria é a previsão de importantes variáveis macroeconômicas, tais como taxas de juros, inflação, e produto interno bruto (PIB). Ainda que as previsões de indicadores econômicos sejam bastante visíveis e, muitas vezes, extensamente publicadas, os métodos econométricos podem ser usados em áreas econômicas que não têm nada a ver com previsões macroeconômicas (WOOLDRIDGE, 2006, p. 1).

A econometria apresenta diversas metodologias, porém o presente texto abordará a análise de séries temporais, especificamente o modelo ARMAX. Serão utilizadas variáveis macroeconômicas como PIB (Produto Interno Bruto), SELIC e Inflação, para a construção de um modelo de previsão do faturamento das 4 empresas siderúrgicas que possuem ações na BM\&FBOVESPA: CSN, Gerdau, Usiminas e Vicunha. As três variáveis macroeconômicas consideradas sofrem forte influência da política fiscal e monetária do Estado. Segundo Moreira (2006), a taxa da SELIC, determinada pelo COPOM (Comitê de Política Monetária) seguindo diretrizes do Banco Central, baliza todas as outras taxas de juros do mercado. A inflação, por sua vez, também sofre influência da política econômica por meio das variações da SELIC e do controle da emissão de moeda. As variações do PIB estão relacionadas à expansão ou retração da economia.

Essas variáveis são bastante influentes no mercado em questão, pois a siderurgia é uma atividade econômica dependente de financiamentos de longo prazo (influenciados pela taxa de juros básica, a SELIC) e alavancada pelo crescimento do mercado o qual é diretamente relacionado com o PIB.

Ao final, são identificados o grau de influência dos faturamentos das empresas estudadas e dos fatores macroeconômicos passados (PIB, SELIC e inflação) sobre o faturamento futuro das empresas, viabilizando assim a possibilidade de mensurar a rentabilidade das empresas por meio das variações dos fatores macroeconômicos.

\section{Previsão e gestão}

Segundo Armstrong (1983), a previsão consiste em estimar eventos futuros objetivando o planejamento e a Tomada de Decisão. Na Administração, as metodologias de projeções são utilizadas com bastante frequência em áreas como Marketing, Finanças e Produção. De acordo com Certo et al. (2005), as técnicas de previsão buscam reduzir as incertezas, maximizando as oportunidades, minimizando as ameaças e possibilitando a criação de planejamentos contingenciais. Existe, no entanto, pouca literatura sobre previsão de faturamento de empresas no Brasil. Em sua maioria, essas previsões se restringem a análises qualitativas e de ambiente externo.

Moraes, Nagano e Merlo (2002) realizaram previsões usando dados de uma rede varejista, na qual os principais objetivos eram vendas, lucratividade e imagem. Foram utilizados modelos de Redes Neurais Artificiais com variáveis endógenas, tais como: faturamento bruto, número de caixas, área de vendas, número de lojas e número de funcionários. O modelo encontrado se mostrou relevante e com boa capacidade de predição.

Hercos Junior (2009) buscou relacionar fatores macroeconômicos (cotação do real em relação ao dólar, inflação, risco Brasil, taxa de juros, balança comercial, PIB brasileiro e mundial) com as seguintes demonstrações contábeis: liquidez corrente, liquidez geral, liquidez seca, composição do endividamento, necessidade de capital de giro. Entretanto, sua análise foi limitada apenas a uma empresa. O estudo conseguiu detectar que os fatores externos foram favoráveis ao setor eletrônico para os anos analisados. 
Na Administração de Empresas, as correlações de variáveis podem ser muito úteis na exploração dos ambientes organizacionais. Existem alguns estudos que procuram identificar essas relações, explicá-las e correlacioná-las com outras variáveis. Beatty e Smith (1987) identificaram aproximadamente sessenta variáveis como determinantes na procura de algum bem a ser adquirido.

Scarpel e Milioni (2001) utilizaram o modelo de regressão Logit para prever a insolvência das empresas estudadas em função dos dados financeiros observados, formulando um modelo estatisticamente significante com $86,7 \%$ de assertividade.

Mendonça, Freitas e Souza (2009) empregaram o método de Mínimos Quadrados Generalizados (MQG) a fim de verificar a influência da Tecnologia da Informação (TI) sobre a produtividade. Neste estudo, os autores apontaram que a TI não representa apenas um diferencial estratégico e sim um fator crítico de sucesso no segmento industrial.

Neste contexto, a metodologia ARMAX oferece a possibilidade de combinar dados passados com variáveis exógenas, úteis para aumentar a capacidade de predição. O uso desta técnica foi exemplificado por Franses (1991), o qual investigou o consumo de cerveja na Holanda. Os objetivos deste estudo foram estimar o consumo de cerveja a partir dos fatores externos como: (temperatura, preço, e gastos dos consumidores). Franses (1991) evidenciou a importância desses fatores sobre a variável dependente consumo de cerveja e, a partir desse mapeamento, formulou estratégias para o equilíbrio da demanda de cerveja.

Os métodos de previsão, que trazem informações à priori, podem ser uma grande aliada na estratégia organizacional, ao gerarem informações essenciais para o estabelecimento de uma gestão eficaz de estratégias.

\subsection{Estratégia}

De acordo com Drucker (2003), a tentativa de predizer não é sinônimo de planejamento, no entanto, a previsão pode ser uma parte do planejamento. A econometria em si não é útil aos administradores se as informações, por ela gerada, não forem convertidas em ações e decisões.

Uma alternativa para transformar as informações em ações é por meio da utilização da metodologia BSC, o Balanced Score Card, ferramenta desenvolvida por Kaplan e Norton (1992) que traduz a visão e a estratégia com objetivos e medidas de desempenho, organizado segundo as perspectivas financeira, dos clientes, dos processos internos e do aprendizado e conhecimento.

Mintzberg, Ahlstrand e Lampel (2000) salienta que a estratégia organizacional pode ser dividida em: Plano, Padrão, Perspectivas, Posição e Ploy. Ao se fazer uma previsão, com base em séries temporais, para definir um curso de ações para o futuro, busca-se, na verdade, dar enfoque à parte da estratégia organizacional na qual o autor classifica como Padrão. Isto ocorre porque os modelos de previsão buscam um padrão de comportamento das séries temporais e correlações observados para definir o futuro das organizações. A "Posição" da empresa no ambiente em que se insere pode ser determinada a partir de um modelo de previsão, o qual prepara a empresa para a realidade com antecedência. Para superar a concorrência (Ploy), é necessário conhecê-la, logo o modelo de previsão deste estudo permite que uma empresa possa estimar os faturamentos futuros das concorrentes e aliar estes dados a pesquisas mercadológicas. Com essas informações, podem-se desenvolver estratégias a partir da captação deste faturamento.

Kotler e Keller (2006) abordam um ponto crucial ao analisarem o macro ambiente, enfatizando a influência dos fatores econômicos e políticos sobre o meio em que a empresa está inserida. Como esses pontos atuam fortemente sobre a estratégia, eles são essenciais para uma análise mais aprofundada de um setor. Assim, a intenção de identificar como esses fatores podem influenciar o macro ambiente em que as empresas do setor siderúrgico se inserem, são determinantes para auxiliar nas exposições ao risco e para delinear as estratégias das empresas.

Desta forma, o presente estudo coletou o faturamento das empresas siderúrgicas a partir do Resultado Bruto, compatível com os Demonstrativos de Resultados do Exercício (DRE). Estes dados foram obtidos dos relatórios financeiros publicados obrigatoriamente pelas empresas de capital aberto, disponíveis no sítio da BM\&FBOVESPA.

\section{Metodologia - ARIMA}

Para a formulação do modelo ARMAX aqui utilizado, é necessário, inicialmente, apresentar uma formulação mais simplista do modelo conhecido como ARIMA. O método de Box e Jenkins (o modelo Autoregressive Integrated Moving Average - ARIMA) consiste na formulação de um processo gerador de dados que segue uma estrutura temporal, utilizando, para isso, apenas a variável dependente e suas respectivas defasagens sem necessariamente incorporar qualquer relação econômica para explicação da variável dependente (FABRIS; COSTA JUNIOR, 2010).

O modelo é basicamente composto pela união de processos Autorregressivos (AR) e de Médias Móveis (MA). Por consequência, o ARIMA (p,d,q) tem a seguinte estrutura (Equação 1),

$$
y_{t}=\alpha+\sum_{i=1}^{p} \beta_{i} y_{t-i}+\sum_{i=1}^{q} \theta_{i} u_{t-i}+u_{t},
$$


em que $y_{t}$ é o processo contemporâneo modelado; $u_{t}$, o resíduo; $\alpha$ é a constante (ou intercepto); $\beta$ e $\theta$ são os coeficientes do termo autorregressivo e de média móvel, respectivamente. $\mathrm{O}$ valor $d$, da estrutura, referencia o número de diferenciações, $y_{t}-y_{t-1}$, realizadas nos dados, usualmente para se atingir estacionariedade. Outra possibilidade é transformar os dados em log retornos, utilizando, para isso, a Equação 2:

$$
y_{t}=\ln \left(\frac{f_{t}}{f_{t-1}}\right) .
$$

Como realçado anteriormente, estas diferenciações são realizadas com o intuito de fornecer séries estacionárias, pressuposto esse necessário para estimação adequada dos parâmetros do modelo ARIMA e, consequentemente, do modelo ARMAX. Segundo Brooks (2008), o processo de modelagem segue os passos de Identificação, Estimação dos parâmetros e Diagnóstico.

O objetivo da identificação é determinar os valores de $p$ e $q$ do modelo ARIMA (p,d,q), ou seja, a forma do processo gerador dos dados. Este processo pode ser realizado por meio da avaliação dos gráficos de autocorrelação e de autocorrelação parcial. Uma ferramenta útil nesta etapa é a criação de correlogramas para investigar o comportamento destas correlações ao longo do aumento das defasagens. Entretanto, estas correlações apenas apontam para a estrutura e não para o número de defasagens exatas. Para tal, são utilizados Critérios de Informações.

Uma vez que o modelo é identificado como representação adequada do mecanismo gerador da série, a estimação dos parâmetros desse modelo é efetuada. Os parâmetros do processo ARIMA podem ser estimados por meio de métodos usuais tais como o de Máxima Verossimilhança (MV), mínimos quadrados (OLS), dentre outros (BROOKS, 2008).

Estimado o modelo, é necessária a verificação do seu ajuste aos dados, o qual, segundo Gujarati (2000), representa checagem averiguação dos pressupostos do modelo, entre eles o fato dos erros amostrais possuírem uma estrutura de Ruídos Brancos (GRANGER; NEWBOLD, 1986). Segundo Brockwell e Davis (2002), a fim de prosseguir com este diagnóstico, faz-se necessário verificar se as autocorrelações amostrais normalizadas dos erros representadas por $\hat{\tau}$ são assintoticamente normais com estrutura igual a (Equação 3):

$$
\hat{\tau}_{k} \sim N(0,1 / T)
$$

em que $\mathrm{T}$ é o tamanho da amostra e $k$ é o lag de defasagem. A verificação de cada lag das autocorrelações pode ser muito dispendiosa. Uma alternativa é a checagem simultânea das autorrelações de 1 a $k \hat{\tau}_{1 \ldots k}$. O teste de hipótese, no qual todos os $k$ lags são iguais a zero, pode ser conduzido pela estatística Q formulada por Ljung e Box (1978), a qual é calculada por (Equação 4):

$$
Q_{L B}=T(T+2) \sum_{j=1}^{q} \frac{\hat{\tau}_{j}^{2}}{T-j} \sim \chi_{q}^{2},
$$

em que T é o tamanho da amostra; $q$ o indexador do lag. A estatística $Q_{\mathrm{LB}}$ sobre a hipótese nula segue uma distribuição $\chi^{2}$ com q graus de liberdade.

Caso a inadequação fique evidenciada, ou seja, a hipótese nula seja rejeitada, o ciclo de identificação, estimação e verificação é novamente aplicado, até que a representação apropriada seja encontrada. Depois da validação do modelo, a previsão dos valores futuros da série temporal modelada pode, enfim, ser obtida.

\subsection{Metodologia-ARMAX}

O modelo ARMAX é um modelo multivariado, pois possibilita a análise de mais de uma variável correlacionada à previsão de $X_{t}$. Segundo Franses (1991), o modelo ARMAX representa a possibilidade de assegurar que todas as variáveis possam, isto é, as variáveis exógenas e as defasagens possam ser consideradas simultaneamente.

O ARMAX é uma extensão do modelo Autorregressivo de Médias Móveis (ARMA) e possui uma variável endógena dependente, que, no presente estudo, é o faturamento e outras variáveis explicativas exógenas. Sua estrutura é apresentada como (Equação 5):

$$
y_{t}=\alpha+\sum_{i=1}^{p} \beta_{i} y_{t-i}+\sum_{i=1}^{q} \theta_{i} \varepsilon_{t-i}+\varepsilon_{t}+\sum_{k=1}^{K} \sum_{j=0}^{w} \delta_{k} x_{t-j, k},
$$

em que $y_{t}$ é o faturamento contemporâneo; $\alpha$ é a constante; e $\beta, \theta, \delta$ são os coeficientes dos termos autorregressivos, da componente aleatória e das variáveis exógenas, respectivamente. Todas as variáveis são indexadas no período de tempo $t$. Os resíduos da regressão têm distribuição, $\varepsilon_{t} \sim N\left(0, \sigma^{2}\right)$.

Os modelos de regressão partem do pressuposto de que as variáveis explicativas são independentes, já o ARMAX defende a dependência das variáveis (mesmo estas sendo endógenas) No mundo financeiro/ econômico e interligado dos dias atuais, sabe-se que é impossível definir variáveis que de fato sejam independentes, em razão disto, o presente estudo optou pela utilização do ARMAX.

Os erros no modelo ARMAX também são tratados de forma diferenciada. Os erros referentes à variável autorregressiva (o faturamento) são estimados segundo as suas defasagens relativas à amplitude da série temporal utilizada no modelo e, também, somados ao erro aleatório referente ao próprio modelo.

A estimação dos parâmetros do modelo ARMAX, pressupõe estacionariedade de suas variáveis, sobre pena de incorrer em um modelo espúrio. Para tal, 
é aplicado o teste de estacionariedade Augmented Dickey-Fuller (ADF). Segundo Bueno (2008), o teste tem como ponto de partida a análise da série a seguir (Equação 6):

$$
\Delta y_{t}=\psi y_{t-1}+\sum_{t=1}^{p} \alpha_{i} \Delta y_{t-i}+\varepsilon_{t},
$$

sendo os erros $\varepsilon_{t}$ não autocorrelacionados. O teste conduz aos mesmos valores críticos de $\psi$, usuais do teste Dickey-Fuller (DF) para a verificação de raiz unitária.

Para encontrar os coeficientes da Equação 5, foram utilizados o método de Máxima Verossimilhança. Nele, foi maximizado o logaritmo natural da seguinte função (Equação 7):

$$
\begin{aligned}
& \ln \left(\varepsilon_{t} \mid \Theta\right)=\frac{T}{2} \log |\Sigma|-\frac{1}{2} \sum_{i=1}^{T}\left(y_{t}-\alpha-\sum_{i=1}^{p} \beta_{i} y_{t-i}-\sum_{i=1}^{q} \theta_{i} \varepsilon_{t-i}-\right. \\
& \left.\sum_{k=1}^{K} \sum_{j=0}^{w} \delta_{k} x_{t-j, k}\right) \Sigma^{-1}\left(-\alpha-\sum_{i=1}^{p} \beta_{i} y_{t-i}-\sum_{i=1}^{q} \theta_{i} \varepsilon_{t-i}-\sum_{k=1}^{K} \sum_{j=0}^{w} \delta_{k} x_{t-j, k}\right),
\end{aligned}
$$

em que $\Theta$ é o espaço paramétrico do ARMAX; $|\Sigma|$ é o determinante da matriz de variâncias e covariâncias.

Para a escolha do melhor modelo, pode-se utilizar o método AIC; segundo Akaike (1973, p. 176), o menor AIC será o modelo de maior confiabilidade. Para diagnosticar o modelo, foi acolhido o critério Akaike Infrmation Criteria (AIC). Como critério de escolha, adotou-se a estrutura que minimize esta estatística, na qual, segundo Akaike (1974), é a estrutura de maior confiabilidade, visto que objetiva-se encontrar o modelo com maior "verossimilhança" (minimizando $-2 \log F V$ ) e menor quantidade de parâmetros (minimizando $2 p$ ). O critério de Informação de Akaike é dado por (Equação 8)

$$
A I C=-2 \log F V+2 p,
$$

em que FV é a Função Verossimilhança do modelo e $p$, o número total de parâmetros. Quanto maior a verossimilhança, menor e melhor o AIC; e quanto mais parâmetros, menos parcimonioso o modelo será, pois, com muitos parâmetros, a previsão se torna cada vez mais precisa, porém menos objetiva e de difícil interpretação.

\section{Resultados empíricos}

A amostra coletada dos faturamentos, das empresas do setor siderúrgico, foi obtida pelo sistema da Bloomberg Data License. Estes dados se referem às receitas brutas realizadas e divulgadas trimestralmente nos relatórios financeiros das companhias. O período compreendeu os anos de 2002 a 2010. As empresas examinadas foram: Companhia Siderúrgica Nacional (CSN); Gerdau S.A.; Metalúrgica Gerdau S.A.; Usinas de Minas Gerais S.A, USIMINAS; Vicunha Siderúrgica S.A. As empresas Gerdau e Metalúrgica Gerdau fazem parte do mesmo grupo empresarial, tendo relatórios financeiros idênticos, logo, a análise do Grupo Gerdau será unificada.

No sítio eletrônico do Instituto de Pesquisa Econômica Aplicada - IPEA (INSTITUTO..., 2011), www.ipeadata.gov.br, foram extraídos os dados macroeconômicos que pautam o estudo. Os indicadores empregados foram: PIB deflacionado para representar a proxy do crescimento da economia do setor; Índice de Preço ao Consumidor Ampliado (IPCA) como medida de inflação; juros da taxa SELIC para figurar o retorno livre de risco do mercado.

Os dados da análise foram dispostos trimestralmente. O tamanho da amostra foi de 35 observações, do $2^{\circ}$ trimestre de 2002 ao último de 2010.

\subsection{Resultados - estacionariedade}

Os gráficos, dispostos no Apêndice 1, das séries temporais, apontam para a estacionariedade dos dados. Os resultados são corroborados pelo teste ADF, o que exemplifica que, após uma ou duas diferenciações, há indícios de que as séries sejam estacionárias. A hipótese de existência de raiz unitária não foi rejeitada a $5 \%$ de confiança para as séries, conforme apresentado na Tabela 1:

O teste de normalidade dos resíduos foi realizado pelo teste Shapiro-Wilk, (FÁVERO; et al., 2009). A hipótese nula assume que os dados seguem uma distribuição Normal. Segundo Reinsel (1997), as variáveis inseridas no ARMAX devem possuir distribuição Normal em razão de os erros

Tabela 1. Teste ADF.

\begin{tabular}{lcccc}
\hline \multicolumn{1}{c}{ EMPRESA } & P-VALOR & $\begin{array}{c}\text { P-valor após } 1^{a} \\
\text { diferenciação }\end{array}$ & $\begin{array}{c}\text { P-valor após } 2^{a} \\
\text { diferenciação }\end{array}$ & $\begin{array}{c}\text { Ordem de } \\
\text { diferenciação }\end{array}$ \\
\hline CSN & 0,0898 & 0,0263 & - & $1^{\mathrm{a}}$ \\
GERDAU & 0,3039 & 0,1281 & 0,0138 & $2^{\mathrm{a}}$ \\
USIMINAS & 0,1662 & 0,0741 & 0,0309 & $2^{\mathrm{a}}$ \\
VICUNHA & 0,9674 & 0,6072 & 0,01 & $2^{\mathrm{a}}$ \\
PIB & 0,1609 & 0,01 & - & $1^{\mathrm{a}}$ \\
SELIC & 0,01 & - & - & 0 \\
INFLAÇÃO & 0,033 & - & - & 0 \\
\hline
\end{tabular}

Fonte: Elaborado pelos autores. 
correlacionados pressupostos possuírem distribuição Normal, o qual só ocorreria se as demais variáveis assim o fossem e para que se justifique a estrutura da função verossimilhança como uma Normal Multivariada usada para a estimação dos parâmetros do modelo.

Para esse estudo, foi empregado um nível de significancia de 5\%, o mesmo utilizado na análise ADF, apresentada anteriormente. $\mathrm{O}$ p-valor dos dados foi de $0,212, \log$, há indícios de que a hipótese nula de normalidade esteja correta.

\subsection{Resultados - ARMAX}

Foi construído um total de 6.480 modelos ARMAX para cada empresa. O método AIC foi determinado para definição do melhor modelo, sendo o de menor AIC o de maior confiabilidade. A Tabela 2 representa a apuração das melhores estruturas avaliadas. Assim, este critério aponta para os modelos com a relação mais apropriada para explicar a retroalimentação do faturamento e também das influências dos fatores macroeconômicos sobre este.

Todos os modelos da empresa Gerdau, utilizam faturamento de cinco períodos anteriores para obter as melhores previsões segundo o critério AIC. Fica claro que, para a Gerdau, os dados passados de até cinco trimestres anteriores são importantes para a projeção das receitas futuras. Também ficou evidenciada a importância dos fatores macroeconômicos e suas defasagens, pois entre os melhores modelos apresentados, todos continham os fatores exógenos e muitas de suas defasagens.

A CSN teve os seus resultados próximos aos da Gerdau. Os melhores modelos de previsão para a CSN incluem de cinco a quatro fatores autorregressivos para prever o faturamento futuro, e as variáveis exógenas apareceram da mesma forma, porém as defasagens da inflação não foram extensas quanto as utilizadas pelos melhores modelos da Gerdau.

A empresa Usiminas, assim como a CSN e a Gerdau, teve muita influência do fator autorregressivo faturamento em suas melhores estruturas. Os fatores macroeconômicos exógenos e suas defasagens também foram frequentes para a organização.

A Vicunha, ao contrário da CSN, teve como melhores modelos aqueles que apresentaram um, dois ou três fatores autorregressivos, e os fatores exógenos, assim como na Gerdau, tiveram as suas defasagens inclusas.

\subsection{Resultados empíricos - avaliação do melhor modelo}

Para que o modelo possa ser analisado pelo ARMAX, os erros devem possuir uma estrutura de independência temporal em torno do zero, pois qualquer padrão que fuja desse formato indicaria omissão de variáveis fundamentais no modelo bem como má especificação do modelo (KOHN, 1979). Depois de encontrada a melhor estrutura especificada na Tabela 2, procedeu-se aos diagnósticos dos MAs de forma a tentar modelar os resíduos como ruídos brancos. O número de defasagem dos erros é sugerido pela estatística Q de Ljung-Box. Os modelos, que excederam 5 lags, não foram avaliados.

\subsubsection{CSN e Vicunha}

A melhor estrutura encontrada para a CSN foi composta por: AR(5), I(1), MA(3), X (PIB, PIB $1: 5$, SELIC, SELIC ${ }_{1: 4}$, Inflação, Inflação $\left.{ }_{1: 4}\right)$, enquanto para a Vicunha AR(3), I(2), MA(3), X (PIB, PIB 1:5, SELIC, SELIC ${ }_{1: 5}$, Inflação, Inflação ${ }_{1: 5}$ ). A verificação dos erros foi realizada para todos os 5 lags, entretanto não foi possível encontrar independência. Desta forma, não se estendeu a análise do ARMAX para as referidas empresas.

\subsubsection{Usiminas}

O melhor modelo sugerido pelo critério AIC para Usiminas é o AR (5), I(2), MA (3), X (PIB, PIB ${ }_{1: 5}$, SELIC, SELIC ${ }_{1: 5}$, Inflação, Inflação ${ }_{1: 5}$ ). Para garantir que o modelo corresponda ao pressuposto de independência dos resíduos, foram feitas análises dos erros. Todavia, o modelo apresentou ruído branco no resíduo com a seguinte estrutura $\operatorname{AR}(5), I(2)$,

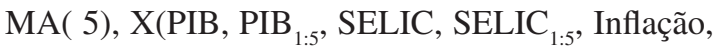
Inflação ${ }_{1: 5}$ ). A estatística $\mathrm{Q}$ indica que a partir do $5^{\circ}$ lag os erros passaram a não possuir mais autocorrelação, com um nível de 5\% de confiança. A Figura 1 ilustra esta e outras relações:

Tabela 2. Melhores modelos de previsão para cada companhia (A simbologia “x:y” ilustra continuidade, por exemplo, PIB 1:5 inclui: PIB.1, PIB.2, PIB.3, PIB.4 e PIB.5 indexado ao respectivo lag.).

\begin{tabular}{|c|c|c|}
\hline EMPRESA & VARIÁVEIS & AIC \\
\hline GERDAU & AR( 5 ),I(2),MA( 3 ), X( PIB,PIB ${ }_{1: 5}$, SELIC,SELIC $_{1: 4}$, Inflação,Inflação $_{1: 5}$ ) & $-234,14$ \\
\hline $\mathrm{CSN}$ & AR( 5 ),I(1),MA( 2 ), X( PIB,PIB ${ }_{1: 5}$, SELIC,SELIC $_{1: 4}$, Inflação,Inflação $\left._{1: 4}\right)$ & $-194,50$ \\
\hline USIMINAS & AR( 5 ),I(2),MA( 1 ), X( PIB,PIB ${ }_{1: 5}$, SELIC,SELIC $_{1: 5}$, Inflação,Inflação $_{1: 5}$ ) & $-203,69$ \\
\hline VICUNHA & AR( 3 ),I(2),MA( 3 ), X( PIB,PIB ${ }_{1.5}$, SELIC,SELIC ${ }_{1 \cdot 5}$,Inflação,Inflação, $)$ & $-132,52$ \\
\hline
\end{tabular}

Fonte: Elaborado pelos autores. 
A Tabela 3 assinala os coeficientes do melhor modelo referente à empresa Usiminas. As receitas brutas anteriores têm uma influência positiva para os próximos períodos. O único erro correlacionado, significante, influi negativamente no modelo de previsão.

Os PIBs se mostraram significantes e positivamente influentes nas receitas futuras. A única defasagem do PIB que apresentou influência negativa é o PIB.5. A taxa SELIC é a variável mais expressiva do modelo, porém não apresenta um padrão, ora é positivamente, ora é negativamente. $\mathrm{O}$ fator inflação não é expressivo quanto às outras variáveis, porém ela tem suas defasagens, exercendo força no sentido positivo no faturamento.

\subsubsection{Gerdau}

O melhor modelo para Gerdau, apresentado pelo AIC, não mostrou independência nos erros. Desta maneira, o MA foi alterado. Assim, a análise para a empresa Gerdau ajustou-se para um AR (5), I (2), MA (1), X (PIB, PIB ${ }_{1: 5}$, SELIC, SELIC 1:4, Inflação, Inflação ${ }_{1: 5}$ ).
A Tabela 4 ilustra as variáveis significantes dos coeficientes aplicados ao melhor modelo referente à empresa Gerdau.

Para a Gerdau, os próprios faturamentos dos dois trimestres anteriores e do quinto trimestre anterior têm uma influência positiva para os próximos, e as receitas de três e quatro trimestres anteriores pressionam negativamente. O único erro significante correlacionado às variáveis dependentes influi positivamente.

O PIB e quase todas as suas defasagens, como era esperado, têm de fato influência positiva nos faturamentos futuros, o que mostra relações intimamente positivas entre as variáveis. A influência do PIB não se apresentou tão expressiva como a da SELIC e da variável dependente.

A SELIC defasada uma vez, como esperado, influi de forma negativa, sendo a variável mais influente do modelo de previsão. Quando ela está defasada três vezes, influi de forma positiva.

As pressões inflacionárias não são tão expressivas quanto das outras variáveis, porém a inflação e suas defasagens afetam em ambos os sentidos o faturamento das empresas analisadas. Este comportamento pode

Standardized Residuals

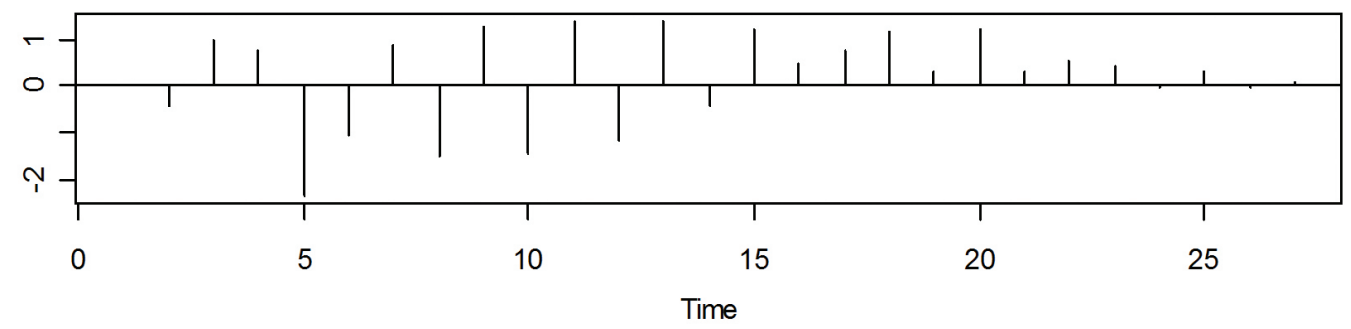

ACF of Residuals

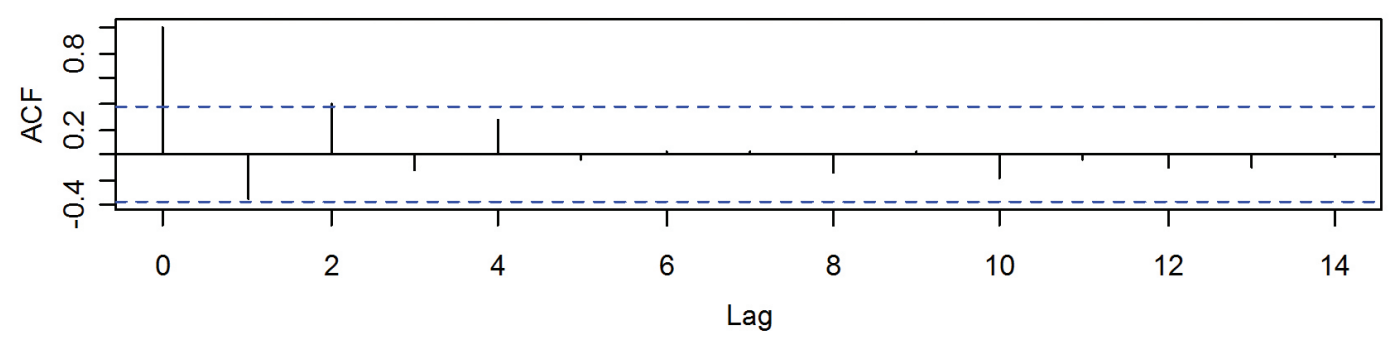

$p$ values for Ljung-Box statistic

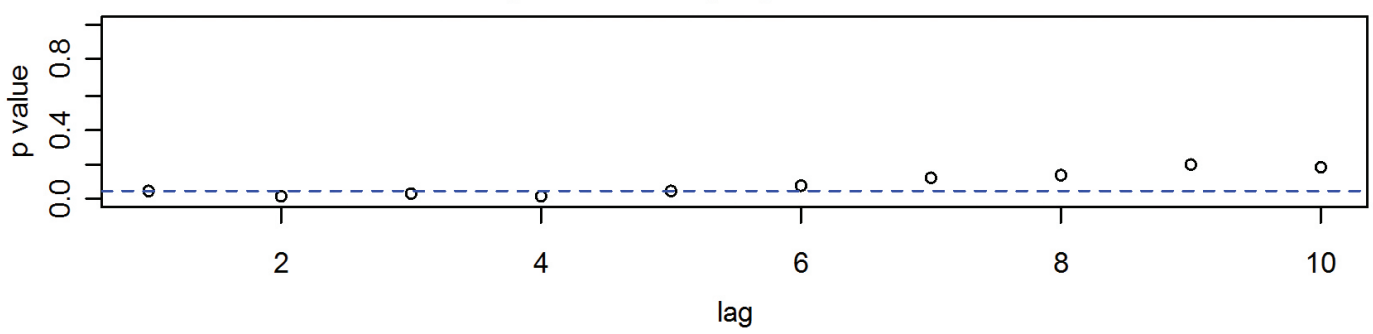

Figura 1. Teste de erros do modelo Usiminas. AR( 5 ), I(2), MA( 5), X( PIB,PIB ${ }_{1: 5}$, SELIC,SELIC $_{1: 5}$, Inflação, Inflação $\left.{ }_{1: 5}\right)$. Fonte: Elaborado pelos autores. 
Tabela 3. Coeficientes Significantes da Usiminas.

\begin{tabular}{cccrc}
\hline & Valor & Std. Error & Z valor & Pr $(>|\mathbf{z}|)$ \\
\hline ar2 & 0,8798 & 0,0072 & 122,0362 & $2,2 \times 10^{-16}$ \\
ar4 & 0,0489 & 0,0082 & 5,9845 & $2,17 \times 10^{-10}$ \\
ma1 & $-0,2916$ & 0,1604 & $-1,8181$ & 0,069053 \\
PIB.0 & 0,3814 & 0,0027 & 139,0537 & $2,2 \times 10^{-16}$ \\
PIB.1 & 0,4715 & 0,0092 & 51,0444 & $2,2 \times 10^{-16}$ \\
PIB.2 & 0,4325 & 0,0138 & 31,3626 & $2,2 \times 10^{-16}$ \\
PIB.3 & 0,4642 & 0,0148 & 31,3421 & $2,2 \times 10^{-16}$ \\
PIB.4 & 0,1689 & 0,0197 & 8,5552 & $2,2 \times 10^{-16}$ \\
PIB.5 & $-0,009$ & 0,0031 & $-2,8804$ & 0,003971 \\
SELIC.1 & $-1,3357$ & 0,1004 & $-13,3090$ & $2,2 \times 10^{-16}$ \\
SELIC.2 & 2,2735 & 0,1407 & 16,1614 & $2,2 \times 10^{-16}$ \\
SELIC.3 & $-1,9126$ & 0,0803 & $-23,8152$ & $2,2 \times 10^{-16}$ \\
SELIC.4 & 2,3738 & 0,0396 & 59,9153 & $2,2 \times 10^{-16}$ \\
INF.0 & 0,1088 & 0,0026 & 41,0747 & $2,2 \times 10^{-16}$ \\
INF.1 & 0,2038 & 0,0064 & 31,6560 & $2,2 \times 10^{-16}$ \\
INF.2 & 0,0494 & 0,0044 & 11,2922 & $2,2 \times 10^{-16}$ \\
INF.3 & 0,1045 & 0,0088 & 11,8508 & $2,2 \times 10^{-16}$ \\
INF.4 & 0,0323 & 0,0055 & 5,8797 & $4,11 \times 10^{-10}$ \\
INF.5 & 0,0134 & 0,0007 & 18,1210 & $2,2 \times 10^{-16}$ \\
\hline
\end{tabular}

Fonte: Elaborado pelos autores.

Tabela 4. Coeficientes significantes da Gerdau.

\begin{tabular}{ccccc}
\hline & Estimativa & Std. Error & Z value & $\operatorname{Pr}(>|\mathbf{z}|)$ \\
\hline ar1 & 1,0269 & 0,0228 & 45,0723 & $2 \times 10^{-16}$ \\
ar2 & 0,5656 & 0,0045 & 126,1069 & $2 \times 10^{-16}$ \\
ar3 & $-0,5508$ & 0,0141 & $-39,1016$ & $2 \times 10^{-16}$ \\
ar4 & $-1,0472$ & 0,0067 & $-155,1951$ & $2 \times 10^{-16}$ \\
ar5 & 0,9785 & 0,0214 & 45,6257 & $2 \times 10^{-16}$ \\
ma1 & 0,8087 & 0,3959 & 2,0424 & 0,0411 \\
PIB.0 & 0,2073 & 0,0004 & 483,7214 & $2 \times 10^{-16}$ \\
PIB.3 & 0,3047 & 0,0013 & 228,4089 & $2 \times 10^{-16}$ \\
PIB.4 & $-0,0075$ & 0,0025 & $-3,0319$ & 0,0024 \\
PIB.5 & 0,0623 & 0,0006 & 102,3515 & $2 \times 10^{-16}$ \\
SELIC.1 & $-2,9359$ & 0,0056 & $-519,8962$ & $2 \times 10^{-16}$ \\
SELIC.3 & 0,6683 & 0,0076 & 87,3800 & $2 \times 10^{-16}$ \\
INF.0 & 0.1164 & 0,0002 & 672,4153 & $2 \times 10^{-16}$ \\
INF.1 & 0,0609 & 0,0010 & 58,5276 & $2 \times 10^{-16}$ \\
INF.2 & $-0,0365$ & 0,0013 & $-29,0276$ & $2 \times 10^{-16}$ \\
INF.3 & 0,0943 & 0,0013 & 72,7950 & $2 \times 10^{-16}$ \\
INF.4 & $-0,02056$ & 0,0003 & $-74,7153$ & $2 \times 10^{-16}$ \\
INF.5 & $-0,0742$ & 0,0007 & $-100,1749$ & $2 \times 10^{-16}$ \\
\hline
\end{tabular}

Fonte: Elaborado pelos autores.

ser explicado pelas expectativas dos Agentes nos ajustes de preços na economia a cada período.

\subsection{Resultados gerais}

De uma forma geral, a variável inflação se mostrou menos expressiva nos modelos e, na maioria das vezes, influi de forma positiva nos faturamentos futuros. Quando este fator exerce pressão negativa, o seu valor é muito baixo ou quase inexpressivo.
A SELIC apresenta as taxas mais expressivas de influência, na maioria das vezes atua negativamente nas receitas futuras. Esta relação se deve, em parte, aos elevados níveis de investimento do setor siderúrgico, pois os aumentos nas taxas de juros desestimulam os agentes a financiarem as atividades e expansão de suas capacidades instaladas.

O PIB apresentou ser menos expressivo que a SELIC. De maneira geral, este se mostrou influindo positivamente na previsão dos faturamentos. 
A hierarquia de influências no modelo de previsão se definiu pela SELIC, seguida dos faturamentos anteriores, PIB e IPCA.

\section{Conclusão}

A previsão é muito importante para um bom planejamento. Neste sentido, o estudo procurou evidenciar um modelo de projeção de receitas que pudesse servir como um instrumento aos gestores, investidores e analistas do mercado.

A presente pesquisa conseguiu apontar para as formas de relações, as melhores estruturas do modelo bem como as forças de influência das variáveis exógenas e do próprio faturamento nos valores futuros de duas entre quatro empresas analisadas com ações na Bolsa de Valores de São Paulo. Entretanto, houve limitação de dados, pois o sítio eletrônico da BOVESPA apenas disponibiliza os dados financeiros das empresas a partir de 2002, o que implicou testes estatísticos menos robustos, uma vez que houve restrição no tamanho amostral.

Um modelo baseado em fatores macroeconômicos pode ser uma ferramenta útil para o orçamento de empresas. Ao pautar-se por dados externos, o ARMAX possibilita aos gestores dimensionar os investimentos, além de oferecer informações sobre outros players de mercado.

Em suma, a pesquisa mostrou, entre outros aspectos, que a Selic é a variável mais influente para os faturamentos do setor siderúrgico, em comparação com o próprio faturamento, o PIB e a Inflação. Tal dado mostra que a taxa de juros, no setor siderúrgico nacional é extremamente importante no modelo de previsão para as empresas Usiminas e Gerdau. Em geral, o PIB e a inflação não foram tão expressivos, embora significantes, ambos influenciam na maioria das vezes positivamente. Esse fato corrobora a hipótese de que o crescimento do País está ligado ao crescimento da indústria e da economia nacional.

Pesquisas futuras, utilizando ARMAX, podem ser realizadas em outros setores de atuação com estruturas diferentes das encontradas no setor pesquisado. Desta forma, será possível auxiliar os agentes de outros segmentos na gestão de seus ativos, na confecção de estratégias organizacionais e proteção contra riscos.

\section{Referências}

AKAIKE, H. A new look at the statistical model identification. IEEE Transactions on Automatic Control, v. 16, n. 6, p. 716-723, 1974. http://dx.doi.org/10.1109/ TAC.1974.1100705

ANDRADE, M. L. A.; CUNHA, L. M. S. O setor siderúrgico. In: BANCO NACIONAL DE DESENVOLVIMENTO ECONÔMICO E SOCIAL - BNDES. BNDES 50 Anos: histórias setoriais. Rio de Janeiro, 2002.

ARMSTRONG, J. T. Strategic planning and forecast fundamentals. Pennsylvania: University of Pennsylvania,
1983. Disponível em: <http://repository.upenn.edu/ cgi/viewcontent.cgi article $=1132 \&$ context $=$ marke ting_papers>. Acesso em: 9 fev. 2013.

BEATTY, S. E.; SMITH, S. M. External search effort: an investigate on across several product categories. Journal of Consumer Research, v. 14, n. 1, p. 83-95, 1987. http://dx.doi.org/10.1086/209095

BROOKS, C. Introductory econometrics for finance. 2nd ed. Cambridge: Cambridge University Press, 2008. http://dx.doi.org/10.1017/CBO9780511841644

BROCKWELL, P. J.; DAVIS, R. A. Introduction to time series and forecasting. New York: Springer Verlag, 2002. http://dx.doi.org/10.1007/b97391

BUENO, R. L. S. Econometria de séries temporais. São Paulo: Cengage Learning, 2008.

CERTO, S. C. et al. Administração estratégica: planejamento e implantação da estratégia. São Paulo: Pearson Education do Brasil, 2005.

DRUCKER, P. Administrando para o futuro: os anos $90 \mathrm{e}$ a virada do século. São Paulo: Cengage Learning, 2003.

FABRIS, T. R.; COSTA JUNIOR, N. C. A. Propriedade das séries temporais dos lucros trimestrais das empresas brasileiras negociadas em bolsa. Revista Brasileira de Finanças, v. 8, n. 3, p. 351-376, 2010.

FÁVERO, L. P. et al. Análise de dados: modelagem multivariada para tomada de decisões. Rio de Janeiro: Elsevier, 2009. 646 p.

FRANSES, P. H. Primary demand for beer in the Netherlands: an application of ARMAX Model Specification. Journal of Marketing Research, v. 28, n. 2, p. 240-245, 1991. http://dx.doi.org/10.2307/3172813

GRANGER, C.; NEWBOLD, P. Forecasting economic time series. Orlando: Academic Press, 1986.

GREMAUD, A. P.; TONETO JÚNIOR, R.; VASCONCELLOS, M. A. S. Economia Brasileira Contemporânea. São Paulo: Atlas, 2005.

GUJARATI, D. N. Econometria básica. São Paulo: Pearson Education do Brasil, 2000.

HERCOS JUNIOR, J. B. Análise de demonstrações contábeis e fatores macroeconômicos. Enfoque: Reflexão Contábil, v. 28, n. 2, p. 9-26, 2009.

INSTITUTO AÇO BRASIL. Relatório de sustentabilidade 2012. Rio de Janeiro, 2013. Disponível em: <http:// www.acobrasil.org.br/site/portugues/biblioteca/ relatorio_sustentabilidade_2012.pdf>. Acesso em: 9 fev 2013.

INSTITUTO DE PESQUISA ECONÔMICA APLICADA - IPEA. Ipeadata macroeconômico. Brasília, 2011. Disponível em: <http://www.ipeadata. gov.br/>. Acesso em: 9 fev. 2013.

KAPLAN, R. S.; NORTON, D. P. The balanced scorecard: measures that drive performance. Harvard Business Review, v. 70, n. 1, p. 71-79, 1992. PMid:10119714.

KOHN, R. Identification results for ARMAX structures. Econometrica, v. 47, n. 5, p. 1295-1304, 1979. http:// dx.doi.org/10.2307/1911964

KOTLER, P.; KELLER, K. Administração de marketing 12. ed. São Paulo: Prentice Hall, 2006.

LJUNG, G. M.; BOX, G. E. P. On a measure of lack of fit in time series models. Biometrika, v. 65, n. 2, p. 297-303, 1978. http://dx.doi.org/10.1093/biomet/65.2.297 
MAXIMIANO, A. C. A. Introdução a administração. São Paulo: Atlas, 2004.

MENDONÇA, M. A. A.; FREITAS, F. A.; SOUZA, J. M. Tecnologia da informação e produtividade na indústria brasileira. Revista de Administração de Empresas, v. 49, n. 1, p. 70-85, 2009. http://dx.doi.org/10.1590/ S0034-75902009000100009

MINTZBERG, H.; AHLSTRAND, B.; LAMPEL, J. Safári de estratégia: um roteiro pela selva do planejamento estratégico. Tradução Nivaldo Montingelli Júnior. Porto Alegre: Bookman, 2000.

MORAES, M. B. C.; NAGANO, M. S.; MERLO, E. M. Previsão de faturamento no varejo brasileiro utilizando-se de um modelo de redes neurais artificiais. In: ENCONTRO NACIONAL DE ENGENHARIA DE PRODUÇÃO, 22., 2002, Curitiba. Anais eletrônicos...
Curitiba: ENEGEP, 2002. Disponível em: <http://www. abepro.org.br/biblioteca/ENEGEP2002_TR71_1085. pdf>. Acesso em: 9 fev. 2013.

MOREIRA, E. M. Política monetária: em 2005, Copom calibra a taxa de juros Selic em busca da meta de inflação. Indicadores Econômicos FEE, v. 33, n. 4, p. 31-40, 2006.

SCARPEL, R. A.; MILIONI, A. Z. Aplicação de modelagem econométrica a análise financeira de empresas. Revista de Administração, v. 36, n. 2, p. 80-88, 2001.

REINSEL, G. C. Elements of multivariate time series analysis. New York: Spinger, 1997. http://dx.doi. org/10.1007/978-1-4612-0679-8

WOOLDRIDGE, J. M. Introdução à econometria: uma abordagem moderna. São Paulo: Cengage Learning, 2006. 


\section{Apêndice 1}

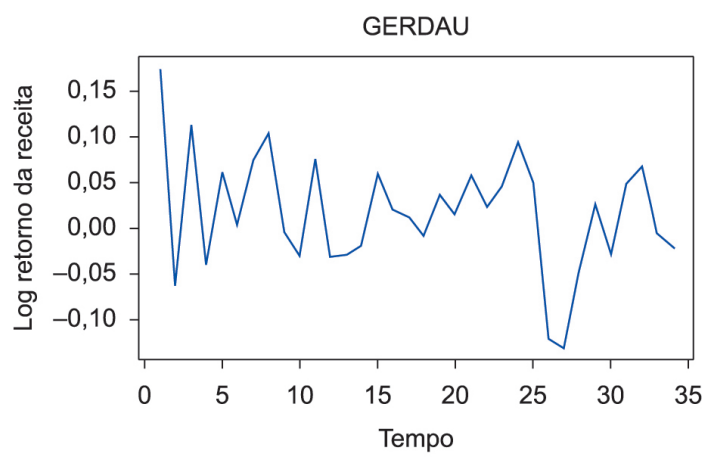

Figura 1. Gráfico da série temporal da Gerdau. Fonte: Elaborado pelos autores.

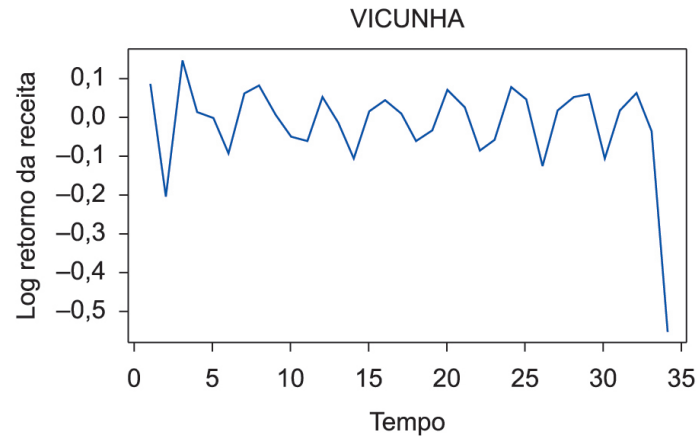

Figura 2. Gráfico de série temporal da Vicunha. Fonte: Elaborado pelos autores.

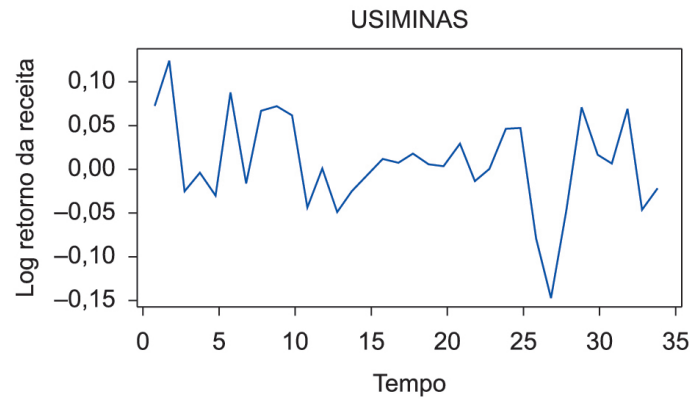

Figura 3. Gráfico da série temporal da Usiminas. Fonte: Elaborado pelos autores.

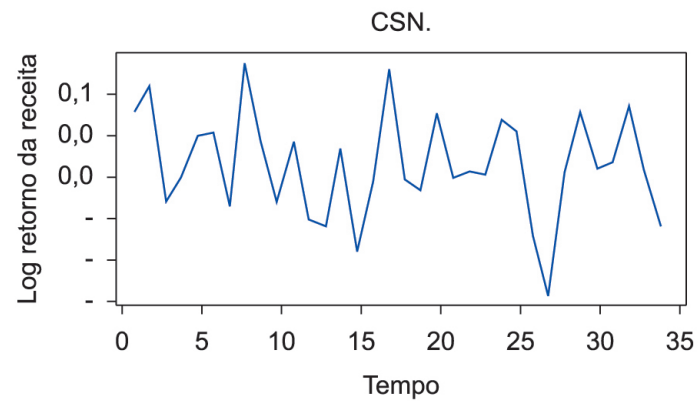

Figura 4. Gráfico da série temporal da CSN. Fonte: Elaborado pelos autores. 


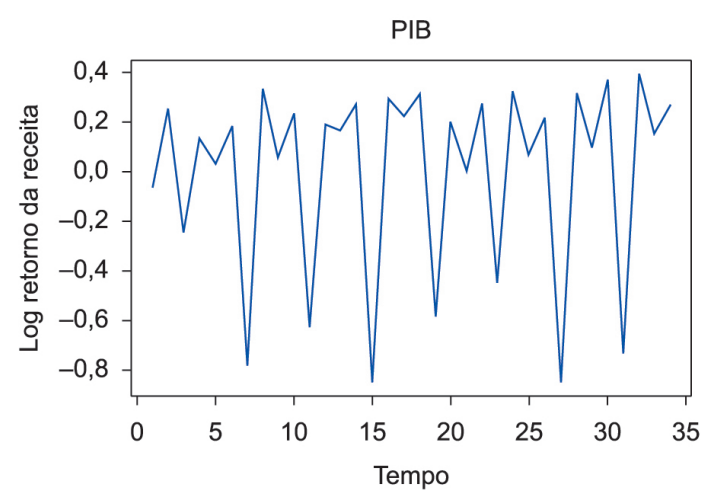

Figura 5. Gráfico da série do retorno do PIB. Fonte: Elaborado pelos autores.

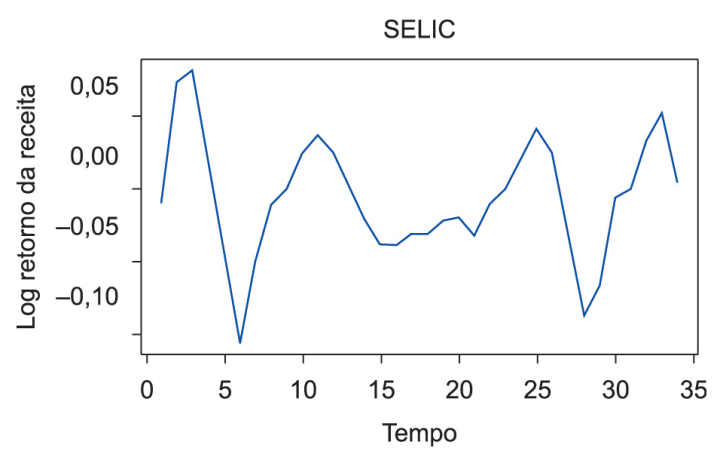

Figura 6. Gráfico da série do retorno da SELIC. Fonte: Elaborado pelos autores.

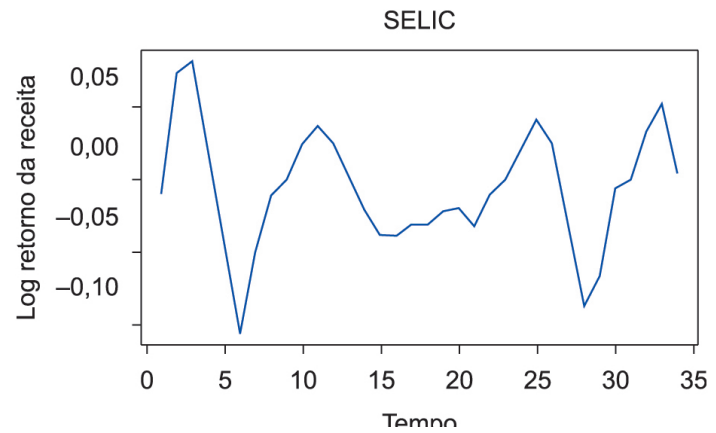

Figura 7. Gráfico da série do retorno da Inflação. Fonte: Elaborado pelos autores. 